\title{
Ambient magnetic fields and dynamos: Pre-main sequence stars and elsewhere
}

\author{
D. Moss ${ }^{\star}$ \\ Department of Mathematics, University of Manchester, Manchester M13 9PL, UK \\ Received 11 August 2003 / Accepted 21 October 2003

\begin{abstract}
The effects of an ambient (i.e. "external") poloidal magnetic field on mean field dynamo action in a sphere are investigated. It is found that an ambient field of strength comparable to that of the poloidal field of the isolated dynamo will inhibit dynamo action. This ambient field will normally be considerably weaker than the typical total field resulting from $\alpha^{2} \omega$ dynamo action. Given earlier similar results for disc dynamos, this appears to be a general result. Applications to pre-main sequence stars and the history of magnetic white dwarfs are discussed.
\end{abstract}

Key words. magnetic fields - stars: evolution - stars: chemically peculiar - stars: magnetic fields - stars: pre-main sequence

\section{Introduction}

Traditional studies of astrophysical dynamos have, quite reasonably, investigated systems subject to boundary conditions that imply the magnetic field tends to zero at large distances from the dynamo-active region, that is that the systems are magnetically isolated. This is true even for models of the solar dynamo with imposed interior fields (e.g. Pudvokin \& Benevolenskaya 1984; Boyer \& Levy 1984; Boruta 1996; see also Moss 1996). More recently, Moss \& Shukurov (2001, 2003) have investigated dynamos in the presence of large scale imposed external magnetic fields, in the context of galactic and accretion discs. A remarkable general result to emerge from this work is that an external field of magnitude of order that of the poloidal field generated by the dynamo in isolation is sufficient to affect strongly the dynamo action.

Moss (2001, 2003a,b) has revisited the long-standing problem of the origin of the magnetic fields of the chemically peculiar (CP) stars of the middle main sequence, paying particular attention to the possibility that large-scale magnetic fields may persist from the ISM to the main sequence, surviving the global Hayashi-phase turbulence expected to be experienced by stars of less than a few solar masses (but see Palla \& Stahler 1993 for an alternative picture of pre-main sequence stellar evolution). One of the possibilities discussed is that a "hybrid" field may be generated, as a result of the interaction between the trapped primordial ("fossil") field and a turbulent dynamo in the pre-main sequence star, either during the initial descent of the Hayashi track when the star is fully convective, or late when a radiative core has developed, and convection occurs in an outer shell.

Moss (2003a,b) attempted to estimate plausible globally averaged poloidal field strengths for a trapped primordial field

\footnotetext{
* e-mail:moss@ma.man.ac.uk
}

as stars of several solar masses contract towards the main sequence. These estimates suggest that these fields may well be about $10 \%$ of the equipartition field strength (although, importantly, fields might locally be much stronger). Given that standard $\alpha^{2} \omega$ mean field dynamos have a total field strength much larger than the poloidal field strength (i.e. $\left|B_{\mathrm{pol}} / B_{\mathrm{tor}}\right|<<1$ ), a priori this suggests that there may be an interesting interaction between fossil and dynamo fields in these pre-main sequence objects.

Thus we investigate the properties of spherical dynamos in the presence of imposed external fields, that are uniform at large distances. Initially we study dynamos in spheres, and extend this to dynamos in spherical shells, partly motivated by the evolution of convective regions as stars evolve towards the main sequence. A somewhat related study, of small scale dynamo action in a region where turbulent convection is driven by an imposed temperature gradient in the presence of an ambient large scale magnetic field, has recently been published by Cattaneo et al. (2003).

\section{The model}

We use a dynamo code based on that described in Brandenburg et al. (1989), solving the standard axisymmetric $\alpha^{2} \omega$ mean field dynamo equations inside the sphere $r=R$, with provision to include nonuniform magnetic diffusivity. The original form of the code imposed boundary conditions at $r=R$ that matched the dynamo field in $r \leq R$ to a vacuum external field, by a procedure equivalent to solving the equations

$\nabla \times \nabla \times \boldsymbol{B}=\mathbf{0}, B_{\phi}=0$,

in the region $r>R$, with the boundary condition that $\boldsymbol{B} \rightarrow \mathbf{0}$ at large distances. Here, this procedure is modified to impose the 
boundary condition that $\boldsymbol{B}_{\mathrm{pol}}=B_{0} \boldsymbol{k}$ at large distances, where $\boldsymbol{k}$ is the unit vector parallel to the axis of symmetry, $\mathrm{O} z$ (in practice, this condition is imposed on the surface $r=f R$, with $f=5$ or 10 ). The condition $B_{\phi}=0$ is retained in $r>R$, i.e. we consider vacuum fields rather than the more general force-free configurations.

We take

$\alpha=\frac{\alpha_{0} a(r) \cos \theta}{\left(1+B^{2} / B_{\mathrm{eq}}^{2}\right)}$,

where $B_{\text {eq }}$ is the equipartition field strength (assumed spatially uniform), and the factor $a(r)$ allows arbitrary radial localization of the alpha-effect. Also $\eta=\eta_{0} \tilde{\eta}(r), \Omega(r)=\Omega_{0} g(r, \theta)$, where $\alpha_{0}, \eta_{0}$ and $\Omega_{0}$ are constants. Note that axisymmetric dynamo solutions are invariant to changes in uniform rotation, and so this rotation law should be regarded solely as prescribing the differential rotation. Thus

$\Delta \Omega=\Omega(R, \pi / 2)-\Omega(0, \pi / 2)=\Omega_{0}(g(R, \pi / 2)-g(0, \pi / 2))$

gives a measure of the absolute differential rotation. An arbitrary uniform rotation $\Omega_{*}$ can be added to $\Omega(r)$, without altering the solution for given $C_{\alpha}, C_{\omega}$. The relative differential rotation is defined as $\Delta \Omega / \Omega_{*}$. Note that, in dimensional units, $\Delta \Omega=\Omega_{0}$.

The equations are nondimensionalized in terms of the length $R$, time $R^{2} / \eta_{0}$ and equipartition field strength $B_{\text {eq }}$, and so in addition to the standard dynamo parameters

$C_{\alpha}=\frac{\alpha_{0} R}{\eta_{0}}, C_{\omega}=\frac{\Omega_{0} R^{2}}{\eta_{0}}$,

there is now the additional parameter $B_{0} / B_{\text {eq }}=B_{\text {ext }}$. Henceforth, dimensionless quantities are used, unless explicitly stated otherwise.

The solutions within the sphere $r=1$ are monitored in terms of three global parameters: the total energy, the ratio $s=$ $E_{\mathrm{pol}} / E_{\mathrm{tor}}$ of the energies in the poloidal and toroidal parts of the magnetic field in $r \leq 1$, and the parity $P=\left(E_{\text {even }}-E_{\text {odd }}\right) / E$ (where $E_{\text {even }}, E_{\text {odd }}$ are respectively the energies in the parts of the field with even ("quadrupolar") and odd ("dipolar") symmetry with respect to the equator, and $E=E_{\text {even }}+E_{\text {odd }}$ ). The mean poloidal field in $r \leq 1$ can then be estimated as $<B_{\text {pol }}>=0.69 \sqrt{s E /(s+1)}$. We also define $q=s^{1 / 2}$, the ratio of the mean poloidal field strength to the mean toroidal field strength in $r \leq 1$.

Because only axisymmetric solutions are considered, the poloidal field can be written as

$\boldsymbol{B}_{\mathrm{pol}}=\nabla \times(A(r, \theta) \widehat{\boldsymbol{\phi}})$,

and we then obtain the standard dynamo equations for $A$ and $B_{\phi}$, including where appropriate the turbulent diamagnetic term $\propto \nabla \eta$.

\section{Results}

\section{1. $\alpha^{2} \omega$ dynamos}

We first investigated a homogeneous dynamo, $a(r)=$ $1, g(r, \theta)=r, \tilde{\eta}(r)=1$, verifying that the new formulation of the outer boundary condition with $B_{\text {ext }}=0$ gave the standard results with a vacuum boundary condition, and in particular that for $C_{\omega}=-10^{4}$, the marginal values of $C_{\alpha}$ were approximately 0.55 and 0.73 for odd and even parity modes, respectively (cf. Roberts 1972).

Then taking $C_{\omega}$ between $-10^{3}$ and $-10^{5}, C_{\alpha}=(1-10)$, we investigated the nature of solutions with unconstrained parity $P$, as $B_{\text {ext }}$ was increased. Results are summarized in Table 1 . In the limit of large $B_{\text {ext }}$, the solutions become indistinguishable from those in the absence of dynamo action $\left(C_{\alpha}=0\right)$ at the same values of $C_{\omega}$ and $B_{\text {ext }}$, with the poloidal field remaining as $B_{\text {ext }} \boldsymbol{k}$ and the toroidal field arising solely from the distortion of this poloidal field by the differential rotation.

A notable feature of these results which appears characteristic of dynamo calculations with an imposed external field is that, as $B_{\text {ext }}$ increases from zero, solutions change from being dynamos slightly modified by the presence of the external field to essentially uniform fields with a small perturbation caused by residual dynamo action, when $\left\langle B_{\text {pol }}>\sim B_{\text {ext }}\right.$ (see also below). Also, this transitional value of $B_{\mathrm{ext}}$ is not significantly different from the value of $\left\langle B_{\mathrm{pol}}>\right.$ for the dynamo solution when $B_{\text {ext }}=0$. Poloidal field structures for $B_{\text {ext }}=0.0,0.01,0.07$ and 0.10 with $C_{\alpha}=1, C_{\omega}=-10^{4}$ are shown in Fig. 1.

In general, as $B_{\text {ext }}$ increases from zero, solutions which are initially singly periodic can be expected to become doubly periodic, before becoming steady as $B_{\text {ext }}$ becomes large (Table 1): until the ambient field is strong enough to dominate the dynamo, it will alternately (every half-cycle) reinforce and weaken the dynamo generated field - cf. also Pudvokin \& Benevolenskaya (1984), Boyer \& Levy (1984). However, the situation appears sometimes more complex (see e.g. Fig. 2). Likewise, pure dynamo solutions that are of even parity $P=+1$ quite naturally pass through mixed parity configurations as $B_{\text {ext }}$ increases. It is perhaps noteworthy that in one case tabulated $\left(C_{\alpha}=3, C_{\omega}=-10^{4}\right.$, Table 1$)$ a dynamo configuration with $P=-1$ when perturbed by an ambient field (with odd parity, of course), experiences a doubly periodic mixed parity episode as $B_{\text {ext }}$ increases through intermediate values.

\section{2. $\alpha^{2}$ dynamos}

We also studied dynamos with $C_{\omega}=0$. The marginal dynamo number for the $\alpha^{2}$ dynamo in a uniform sphere is 7.64, when a dipolar type mode is excited. Results with $C_{\alpha}=10,20$ and a range of values of $B_{\text {ext }}$ are summarized in Table 2. For $B_{\text {ext }} \gtrsim 1$, $q \rightarrow \infty$ and the solutions closely resemble the ambient field configuration in the absence of dynamo action, with no toroidal field and $\boldsymbol{B}=B_{\text {ext }} \boldsymbol{k}$.

\subsection{More results}

Models with a dynamo active "core" surrounded by a lower diffusivity envelope were also computed. Here we took $a(r)=$ $1, \tilde{\eta}(r)=1$ in $r<0.5, a(r)=0, \tilde{\eta}(r)=\eta_{1}<1$ in $r \geq 0.5$, with either $g(r)=1$ everywhere, or $g(r)=0$ in $r \geq 0.5$. In each case there was a narrow transition layer between the inner and outer zones. 

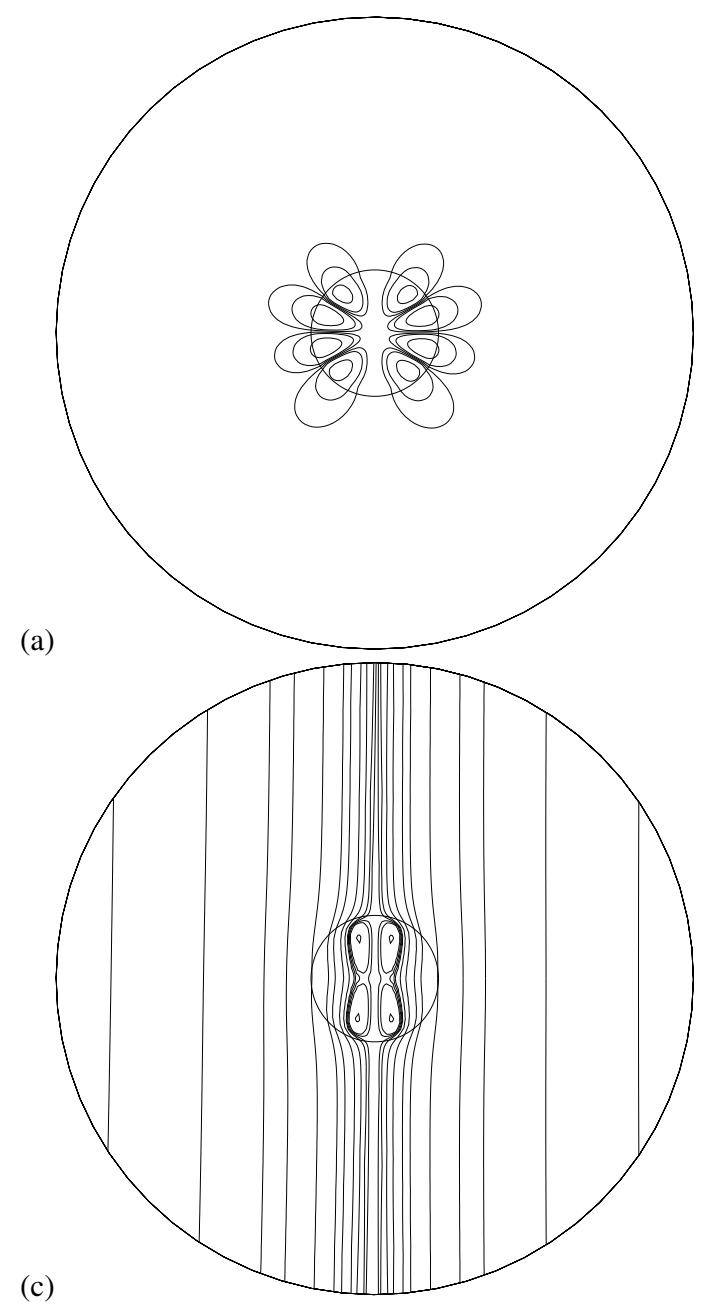

(b)
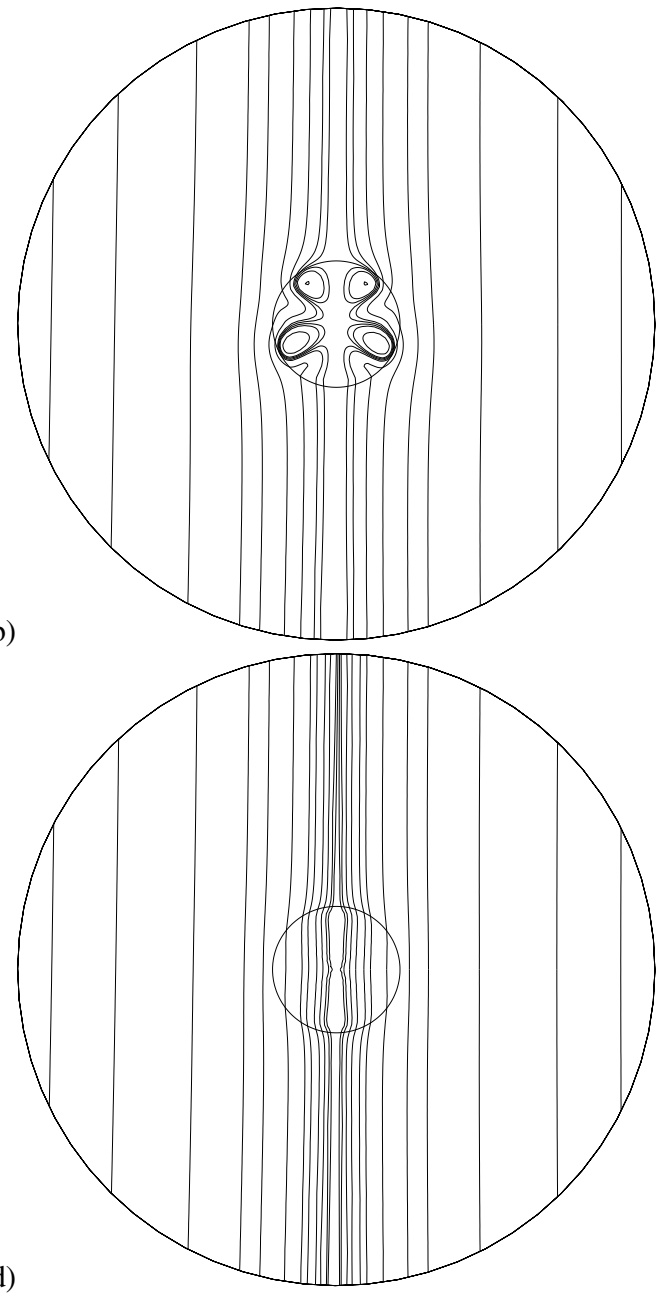

Fig. 1. Poloidal field lines with $C_{\omega}=-10^{4}, C_{\alpha}=1$ and a) $B_{\text {ext }}=0.0$, b) $B_{\text {ext }}=0.01$, c) $B_{\text {ext }}=0.07$ and d) $B_{\text {ext }}=0.10$. The outer circle (sphere) has dimensionless radius 5 , and dynamo action takes place within the inner circle (sphere) $r=1$. Contour levels for the poloidal flux function $2 \pi A r \sin \theta$ are $2 \pi\left(10^{-4}, 3 \times 10^{-4}, 10^{-3}, 2.5 \times 10^{-3}, 6 \times 10^{-3}, 10^{-2}, 2.5 \times 10^{-2}, 6 \times 10^{-2}, 10^{-1}, 0.25,0.60,1.0\right)$, and the maximum values of the contours shown in a)-d) are $2 \pi\left(10^{-3}, 0.10,0.60,1.0\right)$ respectively. Note that a) and $\left.\mathbf{b}\right)$ are snapshots of oscillatory fields.
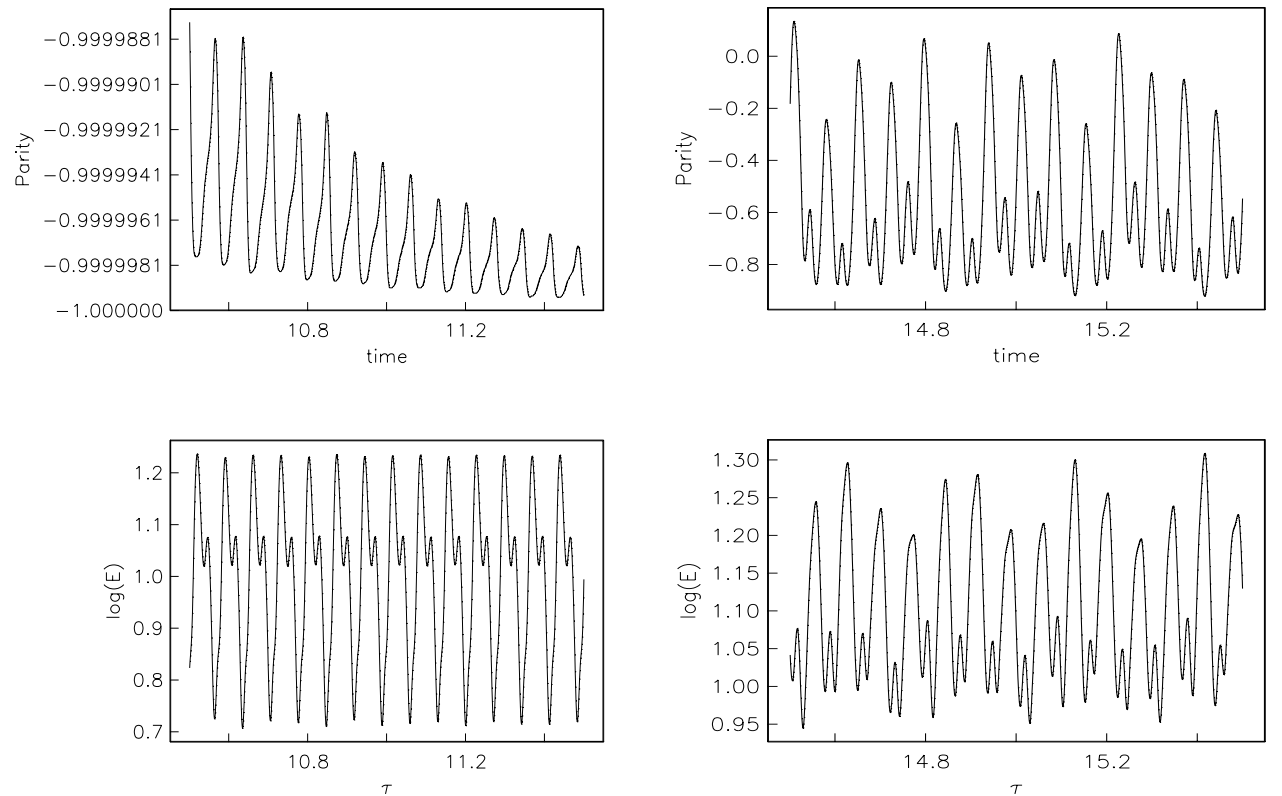

Fig. 2. Parity $P$ and energy $E$ as a function of time for $C_{\alpha}=1, C_{\omega}=-10^{5}$ and $B_{\text {ext }}=0.005$ (left hand panels) and $B_{\text {ext }}=0.01$ (right hand panels). When $B_{\mathrm{ext}}=0.005, P \rightarrow-1$ through a long transient phase (left hand top panel). 
Table 1. Results for $\alpha^{2} \omega$ dynamos in a homogeneous sphere. The values of $\log E,<B_{\text {pol }}>$ and $q$ are global quantities, taken over the sphere $r \leq 1$, and averaged over time for the case of non-steady solutions. In the last column, SP denotes a singly periodic solution, DP doubly periodic and ST a steady solution. When two figures are given in brackets for the parity $P$, this is the range of variation. The entry with $C_{\alpha}=0$ applies to a non-dynamo state, where the external field is amplified solely by the differential rotation.

\begin{tabular}{cccccccc}
\hline \hline$C_{\alpha}$ & $C_{\omega}$ & $B_{\text {ext }}$ & $\log E$ & $q$ & $\left\langle B_{\text {pol }}\right\rangle$ & $P$ & \\
\hline 1 & $-10^{4}$ & 0.0 & -0.28 & $2.3 \times 10^{-2}$ & 0.012 & +1.0 & SP \\
1 & $-10^{4}$ & 0.01 & -0.29 & $2.6 \times 10^{-2}$ & 0.013 & $(+0.72,+0.82)$ & weakly DP \\
1 & $-10^{4}$ & 0.04 & 0.28 & $3.9 \times 10^{-2}$ & 0.028 & -1.0 & ST \\
1 & $-10^{4}$ & 0.10 & 1.80 & $1.5 \times 10^{-2}$ & 0.085 & -1.0 & ST \\
1 & $-10^{4}$ & 1.00 & 3.97 & $1.5 \times 10^{-2}$ & 1.00 & -1.0 & ST \\
0 & $-10^{4}$ & 1.00 & 3.97 & $1.5 \times 10^{-2}$ & 1.00 & -1.0 & ST \\
\hline 1 & $-10^{5}$ & 0.00 & 0.78 & $1.8 \times 10^{-2}$ & 0.030 & +1.0 & SP \\
1 & $-10^{5}$ & 0.01 & 1.12 & $4.7 \times 10^{-3}$ & 0.012 & $(-0.85,+0.20)$ & DP \\
1 & $-10^{5}$ & 0.03 & 2.65 & $1.6 \times 10^{-3}$ & 0.028 & -1.0 & ST \\
0 & $-10^{5}$ & 1.00 & 5.97 & $1.5 \times 10^{-3}$ & 1.00 & -1.0 & ST \\
\hline 10 & $-10^{3}$ & 0.0 & 0.14 & 0.21 & 0.17 & +1.0 & SP \\
10 & $-10^{3}$ & 0.1 & 0.14 & 0.22 & 0.18 & $(+0.78,+0.86)$ & SP \\
10 & $-10^{3}$ & 0.3 & 0.14 & 0.31 & 0.25 & $(-0.29,-0.15)$ & weakly DP \\
10 & $-10^{3}$ & 1.0 & 1.93 & 0.14 & 0.88 & -1.0 & ST \\
\hline 3 & $-10^{4}$ & 0.00 & 0.43 & $3.5 \times 10^{-2}$ & 0.039 & $\approx-1.0$ & SP \\
3 & $-10^{4}$ & 0.01 & 0.44 & $3.5 \times 10^{-2}$ & 0.038 & -1.0 & SP \\
3 & $-10^{4}$ & 0.10 & 0.99 & $3.5 \times 10^{-2}$ & 0.074 & $(-0.90,-0.85)$ & DP \\
3 & $-10^{4}$ & 1.00 & 3.97 & $4.8 \times 10^{-2}$ & 1.00 & -1.0 & ST \\
\hline 10 & $-10^{4}$ & 0.00 & 0.88 & $5.4 \times 10^{-2}$ & 0.10 & +1.0 & SP \\
10 & $-10^{4}$ & 0.10 & 1.18 & $2.0 \times 10^{-3}$ & 0.12 & $(-0.84,+0.12)$ & DP \\
10 & $-10^{4}$ & 1.00 & 3.95 & $1.5 \times 10^{-2}$ & 0.99 & -1.0 & ST \\
\hline & & & & & & &
\end{tabular}

Table 2. As Table 1, but for $\alpha^{2}$ dynamos. In the absence of dynamo action $\left(C_{\alpha}=0\right),\left\langle B_{\mathrm{pol}}\right\rangle=B_{\mathrm{ext}}, q \rightarrow \infty$ and the value of the global magnetic energy is then given by $\log E_{\mathrm{ND}}=0.32+\log B_{\mathrm{ext}}^{2}$.

\begin{tabular}{cccccccc}
\hline \hline$C_{\alpha}$ & $B_{\text {ext }}$ & $\log E$ & $\log E_{\mathrm{ND}}$ & $q$ & $\left\langle B_{\mathrm{pol}}\right\rangle$ & $P$ & \\
\hline 10.0 & 0.0 & -0.70 & $-\infty$ & 1.25 & 0.24 & -1.0 & $\mathrm{ST}$ \\
10.0 & 0.1 & -0.64 & -1.68 & 1.60 & 0.28 & -1.0 & $\mathrm{ST}$ \\
10.0 & 1.0 & 0.35 & 0.32 & 12.9 & 1.03 & -1.0 & $\mathrm{ST}$ \\
\hline 20.0 & 0.0 & 0.19 & $-\infty$ & 1.45 & 0.71 & -1.0 & $\mathrm{ST}$ \\
20.0 & 0.3 & 0.18 & -0.73 & 2.11 & 0.77 & -1.0 & $\mathrm{ST}$ \\
20.0 & 1.0 & 0.43 & 0.32 & 6.85 & 1.12 & -1.0 & $\mathrm{ST}$ \\
\hline
\end{tabular}

Results were of a very similar nature to those described above, allowing for the reduced volume of the dynamo-active region. In particular, the finding that the field was dominated by the external field when $B_{\text {ext }} \gtrsim<B_{\text {pol }}>$ remained. Also, there is no noticeable tendency for the field to be expelled from the dynamo region.

\section{Discussion}

\subsection{General issues}

The clear numerical result is that an imposed external poloidal field with strength in excess of the mean poloidal field of a dynamo in the absence of an imposed field will effectively suppress dynamo action. A similar result has previously been found for mean field dynamos in both accretion discs and galactic discs (Moss \& Shukurov 2001, 2003), which suggests that it is a generic result, at least for dynamos near the $\alpha \omega$ regime. Here it is found to hold for both $\alpha^{2}$ and $\alpha^{2} \omega$ dynamos. In a standard $\alpha^{2}$ dynamo $\left|B_{\text {pol }}\right| \sim\left|B_{\text {tor }}\right|$ (i.e. $s, q \sim 1$ ), so this is not obviously a remarkable result: an external field of magnitude comparable to the poloidal field of the basic dynamo will contribute significantly to the alpha quenching, and so inhibit dynamo action. On the other hand, in an $\alpha^{2} \omega$ dynamo system, the poloidal field is much smaller than the toroidal, and so the total, field $(s, q \ll 1)$ and the result that a value of $B_{\text {ext }}$ such that $|\boldsymbol{B}| \gg B_{\text {ext }} \gtrsim<B_{\text {pol }}>$ is sufficient to inhibit dynamo action may then appear a little surprising at first sight. Two plausible explanations can be advanced.

Firstly, in an $\alpha^{2} \omega$ dynamo system with $\alpha$ set to zero (i.e. no dynamo), differential rotation will generate a strong toroidal field in $r \leq R$ from an imposed poloidal field. If this field is of comparable strength to the equipartition field then alpha quenching from this source alone would severely inhibit any dynamo action. For our models with $C_{\omega}=-10^{3},-10^{4},-10^{5}$ 
and $C_{\alpha}=0$, the ratio $q\left(\propto\left|C_{\omega}\right|\right)$ is approximately $0.15,1.5 \times 10^{-2}$ and $1.5 \times 10^{-3}$ respectively. These values can be compared to those of the corresponding pure dynamo fields (Table 1 with $B_{\text {ext }}=0$ ). Thus an imposed field of strength $B_{\text {ext }}$ of order the value $<B_{\text {pol }}>$ for the pure dynamo field will generate a total field of at least about equipartition strength from winding by differential rotation alone. Only for the entries with $C_{\alpha}=10, C_{\omega}=-10^{3}$, where the effects of differential rotation are becoming smaller, does the value of $q$ marginally exceed these limiting values.

Clearly this is not the complete story, as for intermediate values of $B_{\text {ext }}$ the dynamo and external fields interact in a complex manner (see Fig. 1 and Sect. 3.1). Experience suggests that although mean field dynamos are generally robust with respect to changes in model details, they do need the freedom to selfselect a near-optimal field structure. This freedom will be inhibited by the presence of an imposed poloidal field of strength comparable to that generated by the dynamo. This is hinted at by the entries in Table 1 for intermediate values of $B_{\text {ext }}$, where $<B_{\text {pol }}>$ is smaller than $B_{\text {ext }}$, whereas for larger values of $B_{\text {ext }}$ when dynamo action becomes ineffective, $<B_{\text {pol }}>\rightarrow B_{\text {ext }}$.

For simplicity, only ambient fields parallel to the rotation axis have been considered, maintaing the axisymmetry of the problem. Given the above, to a first approximation it can be predicted that the key parameter of an arbitrarily orientated ambient field will be its component parallel to the vector $\Omega$.

\subsection{Pre-main sequence stars}

In a plausible scenario for the pre-main sequence evolution of what are to become the magnetic CP stars (e.g. Moss 2001, 2003a,b), on the vertical part of the Hayashi track a more-orless fully convective star has formed from, and is immersed in, a medium containing a large scale magnetic field. The estimates in Table 1 of Moss (2003a) suggest that this mean (poloidal) fossil field within the star (and by implication in the immediate environment) may be of order $10 \%$ of the equipartition field strength. The results of Sects. 3.1 and 3.2 above suggest some differences between the effects of an ambient field of strength $\mathrm{O}(0.1) B_{\text {eq }}$ on dynamo action in the two different circumstances of $\alpha^{2} \omega$ and $\alpha^{2}$ dynamo action. If an $\alpha^{2} \omega$ dynamo operates, then it appears that any dynamo action may be significantly inhibited during the Hayashi track evolution of stars of a few solar masses or less. On the other hand, if the stars were to be effectively uniformly rotating, with any dynamo action being of $\alpha^{2}$ type, then external fields of order $B_{\text {eq }}$ are required if significant effects are to occur. The estimates in Moss (2003a) suggest that fossil fields may not be this large (although these estimates are subject to considerable uncertainties, and follow from star formation models that predict that a large fraction of magnetic flux is lost during the formation of a stellar object from the ISM). The difference between the two cases arises because of the absence of conversion of the imposed field to toroidal field by the action of differential rotation in the latter case.

However, a priori it appears unlikely that a gravitationally contracting object would be able to redistribute its angular momentum, via magnetic and turbulent Reynolds stresses, so efficiently that almost uniform rotation could be maintained. Even a small degree of differential rotation can play an important role in dynamo action. Typical rotation periods for pre-main sequence stars of a few solar masses or less seem to be 1-10 days, and mixing length theory estimates of $\eta_{0}$ are of order $10^{13} \mathrm{~cm}^{2} \mathrm{~s}^{-1}$. Then, for example, with the simple rotation law of Sect. 3.1, we get

$$
C_{\omega} \approx 3 \times 10^{6}\left(\frac{\Delta \Omega}{\Omega_{*}}\right) P_{\mathrm{d}}^{-1}\left(\frac{\eta_{0}}{10^{13}}\right)^{-1}\left(\frac{R}{10 R_{\odot}}\right)^{2},
$$

and a relative differential rotation

$$
\left|\frac{\Delta \Omega}{\Omega_{*}}\right|=3 \times 10^{-7}\left|C_{\omega}\right|\left(\frac{\eta_{0}}{10^{13}}\right)\left(\frac{R}{10 R_{\odot}}\right)^{-2} P_{\mathrm{d}},
$$

where $P_{\mathrm{d}}$ is the rotation period in days. Thus with $R=10 R_{\odot}$, $P_{\mathrm{d}}=10 \mathrm{~d}$, even taking $C_{\omega}=-10^{5}$ (i.e. well inside the $\alpha \omega$ regime unless $C_{\alpha} \gg 1$ ) gives a relatively modest $\Delta \Omega / \Omega_{*} \lesssim$ 0.10 . Estimating $C_{\alpha}$ is (even) more uncertain. The classical estimate is $\alpha \sim l \Omega$, where $l$ is the turbulent scale. For the Sun, taking $l=10^{9} \mathrm{~cm}$ gives $\alpha \sim 10^{3} \mathrm{~cm} \mathrm{~s}^{-1}$. However, significantly smaller values have also been derived and used, so we will write $\alpha=f_{\alpha} l \Omega_{*}$, where $f_{\alpha} \leq 1$ represents this uncertainty. Then

$$
\begin{aligned}
\left|C_{\alpha}\right| \approx & 3 \times 10^{4}\left(\frac{l}{5 \times 10^{9} \mathrm{~cm}}\right) \\
& \times f_{\alpha} P_{\mathrm{d}}^{-1}\left(\frac{R}{10 R_{\odot}}\right)\left(\frac{\eta_{0}}{10^{13} \mathrm{~cm}^{2} \mathrm{~s}^{-1}}\right)^{-1}
\end{aligned}
$$

and the ratio $\left|C_{\alpha} / C_{\omega}\right| \approx 5 \times 10^{-3}\left|\Delta \Omega / \Omega_{*}\right|^{-1}(l / 5 \times$ $\left.10^{9} \mathrm{~cm}\right)\left(R / 10 R_{\odot}\right)^{-1}$ is not necessarily small unless $f_{\alpha} \ll 1$ or $\Delta \Omega / \Omega_{*}$ is relatively large. So both $\alpha^{2}$ and $\alpha^{2} \omega$ dynamos should be considered.

If the results of Table 1 for $\alpha^{2} \omega$ dynamos are relevant then the possibility of "hybrid" magnetic fields - that is that any fields surviving to manifest themselves as the fields of the magnetic CP stars (Moss 2001, 2003a,b and references therein) are combinations of fields inherited from the ISM strongly modified by pre-main sequence dynamo action and subsequently frozen in as the majority of the stellar interior becomes stable to convection, near the bottom of the Hayashi track - would largely seem to be eliminated.

If dynamo action on the Hayashi track were to be of $\alpha^{2}$ type with $\left|B_{\text {pol }}\right| \sim\left|B_{\text {tor }}\right|$ (see Table 2), then the situation would be more open, as fossil field strengths may well be less than the local equipartition value (e.g. Moss 2003a). Again, even a relatively weak differential rotation can reduce $q$ significantly (Table 1), so it is unclear how relevant the strictly $\alpha^{2}$ models are in this context.

This discussion has rather ignored the effects of any interaction between the fossil fields and the turbulence in the absence of any possible dynamo action, and in particular the issue of whether the field will be expelled from the turbulent region. It can plausibly be argued that the field becomes concentrated into ropes strong enough locally to control the turbulence, which proceeds more-or-less unimpeded in the field-free regions (see e.g. the discussion in Moss 2003a,b). The effects 
of differential rotation in converting poloidal field to toroidal field with subsequent amplification seem to be unexplored in this context, but appear likely to support the process. The simulations described in Sect. 3 cannot address this issue in detail.

\subsection{CP stars and magnetic white dwarfs}

Turning now to a later stage of stellar evolution, Moss (1989, 2001) estimated possible dynamo field strengths in the convective cores of main sequence A and B stars as being of order $10^{5} \mathrm{G}$. Of course, nothing is known directly about the rotational state of these cores, and whether any such dynamos would be of $\alpha^{2}$ or $\alpha^{2} \omega$ type.

The surface fields (poloidal) of the magnetic CP stars are typically $10^{3}-10^{4} \mathrm{G}$. Again, there is no direct information available about interior field strengths, but even the most recent theoretical models (e.g. Moss 1990), which suggest a relatively modest inward increase of the poloidal field - maybe by a factor of about 10 - predict central poloidal fields of about $10^{4}-10^{5} \mathrm{G}$. These estimates suggest that, even if the ratio $q$ of poloidal to toroidal field strengths in a core dynamo were as large as 0.1 , core dynamos could be significantly affected if not completely inhibited by the envelope field.

Moss (2001) has already pointed out that, if the strongly magnetic white dwarfs are descendants of the magnetic CP stars, as has several times been proposed, then their fields cannot be connected with a core-dynamo field in the near-main sequence CP star ancestors, but must be associated with the anomalously strong fields pervading the radiative envelopes of this group of stars. Given the ubiquity of convective cores on the middle main sequence, the above conclusion follows since if effective core-dynamo action occurs there, whatever the processes that might occur in stellar envelopes to prevent magnetic fields generated by core-dynamos from appearing on the surfaces of the (majority) non-magnetic A and later B stars near the main sequence, all white dwarf precursors could be expected to have central magnetic fields. Thus if the core-dynamo fields are related to the white dwarf fields, it is then difficult to see how all white dwarfs could avoid having broadly comparable fields. This suggests that there is no such relation between white dwarf and main sequence core-dynamo fields, and so any inhibition of a core-dynamo field would not affect the hypothetical link between magnetic white dwarfs and CP stars.

\section{Concluding remarks}

We have demonstrated how, as the ambient field strength is increased from zero, mean field dynamo action in a sphere is at first modified, and then extinguished. The effect of the ambient field becomes significant when its strength is of order that of the poloidal field of the unperturbed dynamo. This result has also been demonstrated in galactic and accretion discs, and we argue that it is universal.

This work has some gross similarities with that recently published by Cattaneo et al. (2003), who studied the effects of an imposed field on turbulent (small-scale) dynamo action, driven in the Boussinesque approximation by an imposed temperature gradient. To quote Cattaneo et al., "there is a gradual change as convection distorts and amplifies the imposed field until its energy eventually exceeds that of the disordered field generated by dynamo action". In the work described above, in the case of $\alpha^{2} \omega$ dynamos the corresponding amplification is by differential rotation. The feedback when the field energy exceeds that of the disordered field is indirectly (and very crudely) modelled by the alpha quenching, in some ways analogous to the assumption that in the small-scale dynamo kinetic and peak magnetic energies are comparable. The similarity between the two rather differently motivated studies arises because in each case the dynamo process is disrupted by feedback caused by amplification of the imposed field by the fluid motions (large-scale here and small-scale in the work of Cattaneo et al.).

Nothwitstanding these similarities, the clear difference is that we here concentrate on the effects of ambient fields on mean field dynamo action, that generates global scale fields.

Acknowledgements. Thanks are due to John Brooke for discussions, and comments on the text.

\section{References}

Boyer, D. W., \& Levy, E. H. 1984, ApJ, 277, 848

Boruta, N. 1996, ApJ, 458, 832

Brandenburg, A., Krause, F., Meinel, R., Moss, D., \& Tuominen, I. 1989, A\&A, 213, 411

Cattaneo, F., Emonet, T., \& Weiss, N. 2003, ApJ, 588, 1183

Moss, D. 1989, MNRAS, 236, 629

Moss, D. 1990, MNRAS, 244, 272

Moss, D. 1996, A\&A, 305, 140

Moss, D. 2001, in Magnetic Fields across the HR Diagram, ed. G. Mathys, S. T. Solanki, \& D. T. Wickramasinghe, ASP Conf. Ser., 248, 305

Moss, D. 2003a, A\&A, 403, 693

Moss, D. 2003b, in Magnetism and Activity of the Sun and Stars, ed. J. Arnaud, \& N. Meunier, EAS Publ. Ser., 9, 21

Moss, D., \& Shukurov, A. 2001, A\&A, 372, 1048

Moss, D., \& Shukurov, A. 2003, A\&A, submitted

Palla, F., \& Stahler, S. W. 1993, ApJ, 418, 414

Pudovkin, M. I., \& Benevolenskaya, Y. Y. 1984, SvA, 24, 458

Roberts, P. H. 1972, Phil. Trans. Phil. Soc. A, 272, 663 